\title{
Diffraction of a Gaussian beam near the beam waist
}

\author{
Evelina A Bibikova ${ }^{1,2+}$, Nazar Al-wassiti ${ }^{2,3 \dagger}$ and Nataliya D Kundikova ${ }^{1,2 *+}$ (i)
}

\begin{abstract}
The behavior of the light field near the beam waist has attracted considerable attention due to the studies of the light spin-orbit interaction: it's manifestation as well as potential applications. Similarly, the light beam diffraction can reveal new light particularities. We have studied the far-field diffraction of a Gaussian beam by a half-plane edge placed near the beam waist. We have solved numerically the Helmholtz equation using the spectral method. We have found symmetric penetration of the light energy into the shadow region if we placed the half-plane edge exactly in the beam waist region. The symmetry gets broken down as the edge moves along the optical axis toward or away from the lens at a distance more than $0.01 \%$ of the focal length. Experimental verification has been obtained using a Gaussian beam focused by a lens of $4 \mathrm{~cm}$ focal length.

The results can be used for precise determination of the beam waist position while investigating the effects of the light spin-orbit interaction in focused Gaussian beams.
\end{abstract}

Keywords: Gaussian beam, Beam waist, Focal plane, Diffraction

\section{Introduction}

The increased interest in the properties of converging light beams is associated with the effects of the spin-orbit interaction of light [1-5]. For the first time, the effect of spin-orbit interaction of light in focused beams was predicted and experimentally observed in a form of a transverse shift of the longitudinal field component at the focal plane when the sign of beam's circular polarization was changed $[6,7]$.

In addition to the transverse, a longitudinal shift from the position of the geometric focus is possible for apertured radially symmetric beams [8]. The displacement of the focus is directed toward the aperture of the light beam and depends on the Fresnel number. For focused Laguerre-Gaussian beams, the longitudinal focal shift increases with increasing focal length but decreases with increasing waist diameter [9].

*Correspondence: kundikovand@susu.ru

† Evelina A Bibikova, Nazar Al-wassiti and Nataliya D Kundikova contributed equally to this work.

${ }^{1}$ Nonlinear Optics Laboratory, Institute of Electrophysics, Ural Branch of the Russian Academy of Sciences, Ekaterinburg, Russia

${ }^{2}$ Department of Optoinformation, South Ural State University, Chelyabinsk, Russia

Full list of author information is available at the end of the article
New effects can be observed under the light diffraction by a straight edge. Optical vortices arise if a beam without optical vorticity diffracts [10] The diffraction of polarized radiation by a straight edge made it possible to determine the dependence of the diffraction pattern on the sign of the circular polarization, and also to demonstrate the vortex character of the longitudinal component of the circularly polarized radiation [11].

The diffraction of Gaussian beams with the optical vorticity by the straight edge leads to the appearance of polarization singularities caused by the longitudinal and transverse components of the electric field of the diffracted beam [12]. The diffraction of light beams by the straight edge makes it possible to determine the direction of the energy flow in beams with optical vortices $[13,14]$.

There are a lot of studies devoted to the edge beam diffraction [11, 12, 15-17] and to the diffraction of focused light beams by different apertures [18-21]. Only few papers are dealing with edge diffraction of focused beams. It was shown that a lens focuses the light beam diffracted by the straight edge into a line-shaped pattern perpendicular to the straight edge [22]. The diffraction of focused radiation by a straight edge may provide new information on the behaviour of radiation in the waist region. Here we present our results of the study of the

\section{Springer Open}

(c) The Author(s). 2019 Open Access This article is distributed under the terms of the Creative Commons Attribution 4.0 International License (http://creativecommons.org/licenses/by/4.0/), which permits unrestricted use, distribution, and reproduction in any medium, provided you give appropriate credit to the original author(s) and the source, provide a link to the Creative Commons license, and indicate if changes were made. 

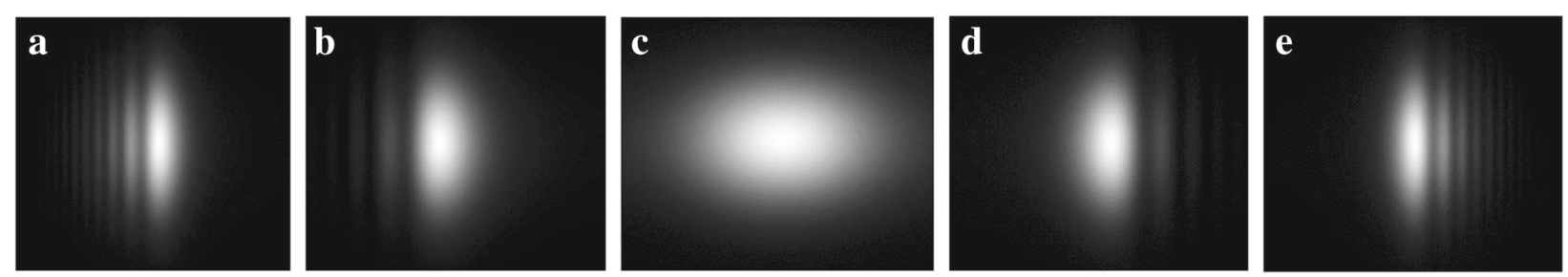

Fig. 1 Calculated far-field intensity distributions. The screen is between the lens and the focal plane (a, b); at focal plane (c); after the focal plane (d, f)

focused Gaussian beam diffraction by a straight edge placed in the beam waist region.

\section{Methods}

To numrically investigate the focused Gaussian beam diffraction by the edge of the screen placed near the focal plane, we used the following procedure. At the input plane $(z=0)$, we described the light field of the focused Gaussian beam as follows

$$
E(x, y, z=0)=E_{0} \exp \left(-\frac{x^{2}+y^{2}}{r_{0}^{2}}\right) \exp \left(i \frac{x^{2}+y^{2}}{f}\right) .
$$

Here $r_{0}$ is the beam radius, and the coefficient $f$ is connected with the focal plane position. Using fast Fourier transform we decomposed the input light field into a set of plane waves and solved the Helmholtz equation for each plane wave at a distance $L$. Using inverse Fourier transform we obtained the light field distribution $E_{L}(x, y, z=L)$ at a given distance $L$ before, at, or after the focal plane. To model beam diffraction by straight edge placed at the distance $L$ from the lens, we used Gaussian beam field $E_{L}$ multiplied by the Heaviside step function as shown in Eq.(2):

$$
E_{\mathrm{D}}(x, y, z=L)=E_{L}(x, y, z=L) \vartheta_{x}(x, y) .
$$

Here $\vartheta_{x}(x, y)$ is the Heaviside step function given by

$$
\vartheta_{x}(x, y)= \begin{cases}1 & x \leq 0 \\ 0 & x>0\end{cases}
$$

To get the far field intensity profile, we used Fourier transform of $E_{\mathrm{D}}(x, y, z=L)$ and determined the intensity of the field profile $I_{\mathrm{D}}(x, y, z=\infty)$. The wavelength of the light was $\lambda=632.8 \mathrm{~nm}$.

To investigate the diffraction of a focused Gaussian beam experimentally, we used the radiation of a $\mathrm{He}-\mathrm{Ne}$ laser generated on the main transverse mode at the wavelength $\lambda=632.8 \mathrm{~nm}$. A lens with the focal length of $4 \mathrm{~cm}$ focused the laser beam. An opaque screen with a vertical edge was mounted on a two-coordinate micromotion stage. We can smoothly insert the screen into the beam, overlapping the right half of the beam, and move the screen along the beam propagation. We recorded the diffraction patterns in the far field by a CCD camera.

\section{Results and discussion}

Figure 1 shows the calculated diffraction patterns intensity distribution for different positions of the screen. We can see that the diffraction pattern changes with the screen move along the beam propagation axis. If the screen is located in the waist region, the intensity distribution is symmetric with respect to the screen edge and forms
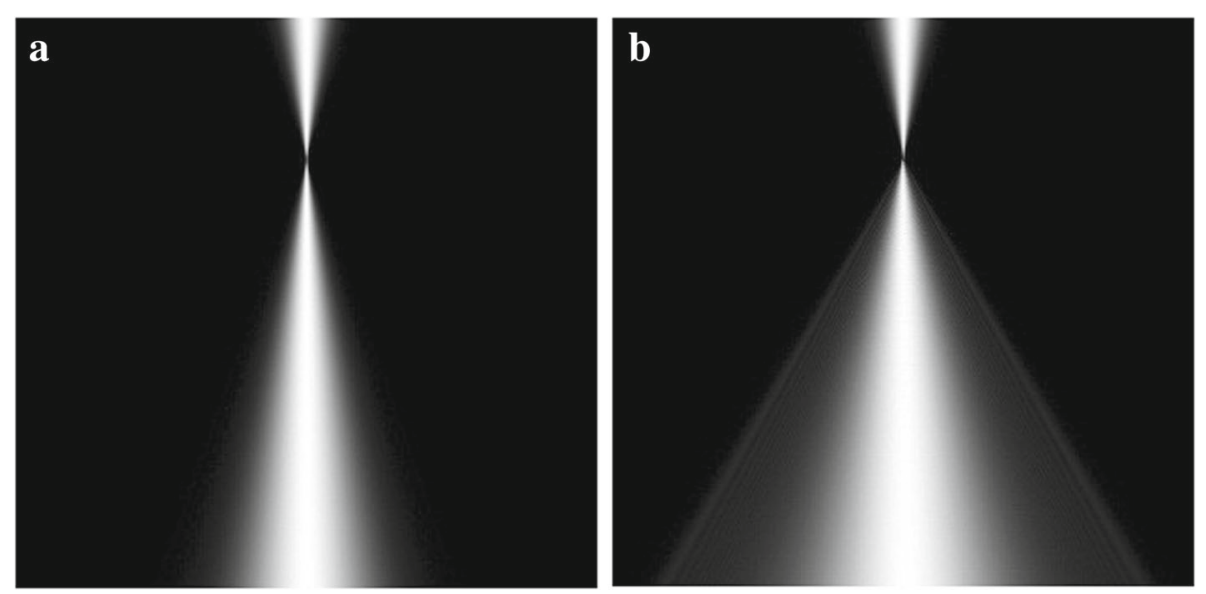

Fig. 2 The evolution of the focused Gaussian beam intensity profiles taken along the beam diameter as a function of distance: (a) the screen is removed from the beam; $(\mathbf{b})$ the screen is located at the focal plane 

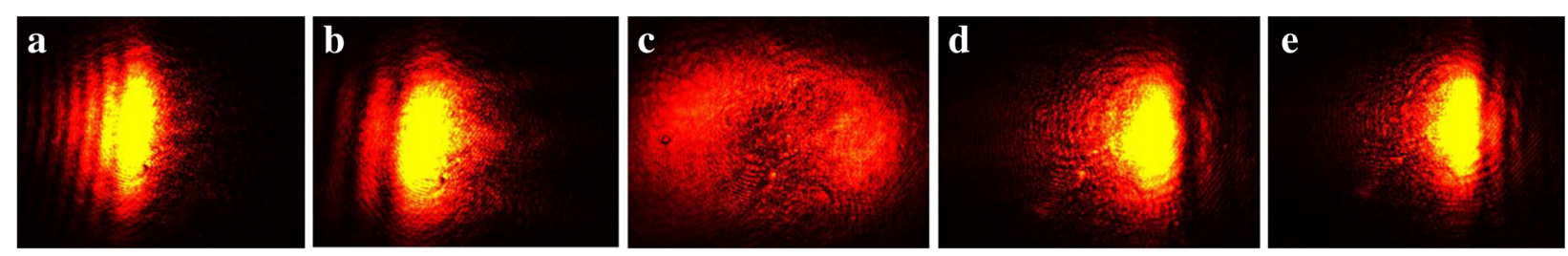

Fig. 3 The experimentally obtained diffraction patterns in the far field at the different distance from the focal plane: a) the screen is located at the distance of $1.5 \mathrm{~mm}$ before the focal plane, $\mathbf{b}$ ) the screen is located at the distance of $75 \mu \mathrm{m}$ before the focal plane, $\mathbf{c}$ ) the screen is located in the focal plane, d) the screen is located at the distance $75 \mu \mathrm{m}$ after the focal plane, e) the screen is located at the distance of $1.5 \mathrm{~mm}$ after the focal plane

an ellipse, the major ellipse axis is perpendicular to the screen edge (Fig.1c). The symmetry gets broken down as the half-plane edge moves along the optical axis toward or away from the lens at a distance more than $0.01 \%$ of the focal length, while the intensity distribution has an evident diffraction pattern and forms a semicircle with diffraction fringes. If the screen is far from the beam waist, the diffraction pattern is more evident, and the diffraction pattern image depends on the screen location (before or after the waist region). If the screen is located before the beam waist, the diffraction fringes are to the left of the screen edge (Fig. 1a, b). If the screen is located after the beam waist, the diffraction fringes are to the right of the screen edge (Fig. 1d, f).

To underline the specific character of the Gaussian beam diffractions at the screen edge located in the focal plane, we calculated transverse light intensity distribution along the diffracted beam propagation. Figure 2 shows the evolution of the focused Gaussian beam intensity profiles, taken along the beam diameter as a function of distance obtained by numerical simulations (Fig. 2a) and for the evolution of the focused Gaussian beam intensity profiles after diffraction at the opaque screen edge placed at the focal plane (Fig. 2b). One can see from Fig. 2 that the beam intensity profiles after the diffraction are symmetrical and wider comparing the focused Gaussian beam intensity profiles.

Figure 3 shows diffraction patterns experimentally recorded under the different screen position relative to the focal plane, namely, the screen is located before the focal plane at the distance of $1.5 \mathrm{~mm}$ and $75 \mu \mathrm{m}$, the screen is located in the focal plane, the screen is located after the focal plane at the distance of $75 \mu \mathrm{m}$ and $1.5 \mathrm{~mm}$. Figure 3 shows the diffraction patterns change under the screen move along the direction of the beam propagation. We can see vertical diffraction fringes in the left part of the diffraction pattern at certain positions of the screen (Fig. 3a, b). At the focal plane, the diffraction pattern has the ellipse form, and the major ellipse axis is perpendicular to the screen edge (Fig. 3c). As the screen move in the direction of the beam propagation, vertical diffraction fringes appear in the right part of the diffraction pattern (Fig. 3d, e).
The experimental investigations have shown that moving the screen at the distance of $20 \mu \mathrm{m}$ from the focal waist breaks the symmetry of the diffraction pattern and the fringes appear. Such diffraction pattern behaviour allows us to indicate the beam focal waist position. We were successful to determine the beam focal waist position using the lens with the focal length of $4 \mathrm{~cm}$ with the accuracy $\pm 20 \mu \mathrm{m}$.

\section{Conclusions}

We have investigated the focused Gaussian beam diffraction by the straight edge in the vicinity of the beam waist. We have observed that the diffraction pattern becomes symmetrical relative to the straight edge if the screen is placed in the beam focal waist. The results can be useful for the precise determination of the beam focal waist position.

\section{Acknowledgements \\ Not applicable.}

\section{Authors' contributions}

All authors made contributions to this work

Funding

Not applicable.

Availability of data and materials

All data are available in the present paper.

Competing interests

The authors declare that they have no competing interests.

\section{Author details \\ ${ }^{1}$ Nonlinear Optics Laboratory, Institute of Electrophysics, Ural Branch of the Russian Academy of Sciences, Ekaterinburg, Russia. ${ }^{2}$ Department of Optoinformation, South Ural State University, Chelyabinsk, Russia. \\ ${ }^{3}$ Department of Physics, College of science, Al-Mustansiriyah University, Baghdad, Iraq}

Received: 4 March 2019 Accepted: 16 July 2019

Published online: 29 July 2019

\section{References}

1. Dugin, A. V., Zel'dovich, B. Y., Kundikova, N. D., Liberman, V. S.: Effect of circular polarization on the propagation of light through an optical fiber. JETP Lett. 53(4), 197-199 (1991)

2. Dooghin, A. V., Kundikova, N. D., Liberman, V. S., Zeldovich, B. Y.: Optical Magnus effect. Phys. Rev. A. 45(11), 8204-8208 (1992)

3. Liberman, V. S., Zel'dovich, B. Y.: Spin-orbit interaction of a photon in an inhomogeneous medium. Phys. Rev. A. 46(8), 5199-5207 (1992) 
4. Bliokh, K. Y., Rodríguez-Fortuño, F. J., Nori, F., Zayats, A. V.: Spin-orbit interactions of light. Nat. Photon. 9(12), 796-808 (2015)

5. Abdulkareem, S., Kundikova, N.: Joint effect of polarization and the propagation path of a light beam on its intrinsic structure. Opt. Express. 24(17), 19157-19165 (2016). http://arxiv.org/abs/1604.03371

6. Baranova, N. B., Savchenko, A. Y., Zel'dovich, B. Y.: Transverse shift of a focal spot due to switching of the sign of circular polarization. JETP Lett. 59(1), 232-234 (1994)

7. Zel'dovich, B. Y., Kundikova, N. D., Rogacheva, L. F.: Observed transverse shift of a focal spot upon a change in the sign of circular polarization. JETP Lett. 59(1), 766-769 (1994)

8. Nicola, S. D., Anderson, D., Lisak, M.: Focal shift effects in diffracted focused beams. Pure Appl. Opt. Part A. 7(1), 1249-1259 (1998)

9. Ren, Z. C., Qian, S. X., Tu, C., Li, Y., Wang, H. T.: Focal shift in tightly focused Laguerre-Gaussian beams. Opt. Commun. 334(1), 156-159 (2015)

10. Zeylikovich, I., Nikitin, A.: Diffraction of a gaussian laser beam by a straight edge leading to the formation of optical vortices and elliptical diffraction fringes. Opt. Commun. 413(1), 261-268 (2018)

11. Bekshaev, A. Y.: Spin-orbit interaction of light and diffraction of polarized beams. J. Opt. 19(8), 085602 (2017)

12. Luo, Y., Lu, B.: Polarization singularities of Gaussian vortex beams diffracted at a half-plane screen beyond the paraxial approximation,. J. Opt. Soc. Am. A. 26(9), 1961-1966 (2009)

13. Terborg, R. A., Volke-Sepulveda, K.: Quantitative characterization of the energy circulation in helical beams by means of near-field diffraction. Opt. Express. 21(3), 3379-87 (2013)

14. Kundikova, N., Popkov, I.: Difraction by a slit hollow chain-like beams with a wavefront dislocation. Russ. Phys. J. 58(1), 61-63 (2015)

15. Bekshaev, A. Y., Mohammed, K. A.: Spatial profile and singularities of the edge-diffracted beam with a multicharged optical vortex. Opt. Commun. 341(1), 284-294 (2015)

16. Worku, N. G., Gross, H.: Propagation of truncated Gaussian beams and their application in modeling sharp-edge diffraction. J. Opt. Soc. Am. A. 36(5), 859 (2019)

17. Bekshaev, A., Chernykh, A., Khoroshun, A., Mikhaylovskaya, L.: Singular skeleton evolution and topological reactions in edge-diffracted circular optical-vortex beams. Opt. Commun. 397(1), 72-83 (2017)

18. Li, Y., Wolf, E.: Focal shifts in diffracted converging spherical waves. Opt. Commun. 39(4), 211-215 (1981)

19. Givens, M. P.: Focal shifts in diffracted converging spherical waves. Opt. Commun. 41(3), 145-148 (1982)

20. Herman, R. M., Pardo, J., Wiggins, T. A.: Diffraction and focusing of Gaussian beams. Appl. Opt. 24(9), 1346 (1985)

21. Bischoff, J., Neundorf, W.: Effective schema for the rigorous modeling of grating diffraction with focused beams. Appl. Opt. 50(16), 2474 (2011)

22. Livanos, A. C., George, N.: Edge Diffraction of a Convergent Wave. Appl. Opt. 14(3), 608 (1975)

\section{Publisher's Note}

Springer Nature remains neutral with regard to jurisdictional claims in published maps and institutional affiliations.

\section{Submit your manuscript to a SpringerOpen ${ }^{\circ}$ journal and benefit from:}

- Convenient online submission

- Rigorous peer review

- Open access: articles freely available online

- High visibility within the field

- Retaining the copyright to your article

Submit your next manuscript at $\gg$ springeropen.com 